\title{
Stability Analysis on the Initial Surface-Wave Generation with an Air-Sea Coupled Shear Flow
}

\author{
Wu-Ting Tsai \\ Professor of Institute of Hydrological Sciences, National Central University, Jung Li, Taiwan 320; and Department of \\ Civil Engineering, National Chiao Tung University, Hsin Chu, Taiwan 300., wttsai@cc.ncu.edu.tw \\ Mei-Ying Lin \\ Ph.D. candidate of Department of Civil Engineering, National Chiao Tung University, Hsin Chu, Taiwan 300.
}

Follow this and additional works at: https://jmstt.ntou.edu.tw/journal

Part of the Civil and Environmental Engineering Commons

\section{Recommended Citation}

Tsai, Wu-Ting and Lin, Mei-Ying (2004) "Stability Analysis on the Initial Surface-Wave Generation with an Air-Sea Coupled Shear Flow," Journal of Marine Science and Technology. Vol. 12: Iss. 3, Article 9.

DOI: $10.51400 / 2709-6998.2239$

Available at: https://jmstt.ntou.edu.tw/journal/vol12/iss3/9

This Research Article is brought to you for free and open access by Journal of Marine Science and Technology. It has been accepted for inclusion in Journal of Marine Science and Technology by an authorized editor of Journal of Marine Science and Technology. 
Stability Analysis on the Initial Surface-Wave Generation with an Air-Sea Coupled Shear Flow

Acknowledgements

This work was supported by grants from the National Science Council of Taiwan (NSC 91-2611-M008-002 and 92-2611-M-008-003). 


\title{
STABILITY ANALYSIS ON THE INITIAL SURFACE-WAVE GENERATION WITHIN AN AIR-SEA COUPLED SHEAR FLOW
}

\author{
Wu-Ting Tsai* and Mei-Ying Lin**
}

Key words: stability analysis, wind waves, air-sea interaction, shear flow.

\section{ABSTRACT}

The temporal instability of a two-dimensional perturbed wave in a coupled air-water shear flow is considered to study the generation and initial growth of wavelets by the air flow. A robust numerical method is developed to solve the coupled Orr-Sommerfeld equations and the interfacial conditions governing the linear viscous instability of the perturbed flow. Calculations of the growth rates and phase speeds of the unstable wavelets compare well with the early theoretical as well as numerical predictions, and also reveal that the discrepancy in the numerical results of Wheless and Csanady (1993) indeed are due to erroneous calculations. Dependence of the instability on the flow parameters is then studied systematically. Contrary to the previous findings, there is no apparent correlation between the maximum growth rate and the minimum phase velocity wavenumber.

\section{INTRODUCTION}

The initial surface-wave generation and growth triggered by the action of wind is the most well-known and obvious example of air-sea interaction. The subject has been intensively studied since the pioneering theoretical model proposed by, e.g., Phillips (1957), Benjamin (1959), and Miles (1957a). Nevertheless, neither of the predicted growth rates by these models, in particular those for short wind waves in gravity-capillary region, agrees with the experimental measurements. It was not until the analysis of Miles (1962), in which the instability of an air shear flow over the water surface was considered, that reasonable growth rates for gravity-capillary waves were obtained. Miles's analysis did not include the shear flow in the water; it is known that the phase speeds of wind-generated gravity-capillary waves depend on the wind-induced drift in the

Paper Submitted 05/17/04, Accepted 06/03/04. Author for Correspondence: Wu-ting Tsai.E-mail:wttsai@cc.ncu.edu.tw.

*Professor of Institute of Hydrological Sciences, National Central University, Jung Li, Taiwan 320; and Department of Civil Engineering, National Chiao Tung University, Hsin Chu, Taiwan 300.

**Ph.D. candidate of Department of Civil Engineering, National Chiao Tung University, Hsin Chu, Taiwan 300. water. Thus the analysis was further improved by taking into account the shear flow in the water by, e.g., Valenzuela (1976), Kawai (1979), and Wheless and Csanady (1993).

Valenzuela (1976) was among the first who studied the growth of the instability waves on the air-sea interface by solving numerically the full formulation of the Orr-Sommerfeld equation and the interfacial conditions for the coupled air-water shear flow Lock (1954). The numerical results of Valenzuela (1976) demonstrated that the shear flow in the water can produce a significant increase in the growth of wind-generated gravity-capillary waves. Later Kawai (1979) carried out intensive studies on the initial generation and evolution of the instability waves both through theoretical analyses and laboratory measurements. He found that the frequencies, the growth rates and the phase velocities of the most unstable waves from the stability analysis are virtually coincident with those of the observed initial wavelets in the laboratory experiments. He therefore concluded that the generation of the initial wavelets at the air-sea interface is caused by selective amplification of the most unstable waves in the coupled airwater shear flow.

Wheless and Csanady (1993) returned to the full formulation of the instability wave problem, the same as that in the investigations of Valenzuela (1976) and Kawai (1979), with focuses on studying the internal structure of the instability waves and also extending the range of flow parameters explored. They developed a numerical technique to integrate the coupled OrrSommerfeld equation based on the compound matrix method, a numerical technique which combines the inviscid and viscid solutions through the use of Ricatti transformation. They, nevertheless, claimed that the developed computational method could not cope with the near-discontinuity in the second derivative of the mean air-flow profile characterizing the linear-logarithmic distribution used in most of the previous investigations. For this reason they constructed a new smooth mean velocity profile with a continuous second derivative. The calculated growth rates that Wheless 
and Csanady (1993) obtained, however, are almost a factor of two larger than those calculated by Kawai (1979) who used linear-logarithmic air velocity profile. They therefore attributed the large discrepancies to the differences in velocity profiles. To clarify the peculiar results obtained by Wheless and Csanady (1993), we decided to reappraise the problem by first developing a robust and accurate numerical method for stability analysis of the air-water coupled shear flow. The numerical implementation is then used to explore the dependence of the generation of instability waves on external flow properties.

Unlike the single-phase stability problem in which the Reynolds number is the only governing parameter, the formulation of the two-phase stability problem requires at least six dimensionless parameters. Due to this complexity, previous studies usually apply to a limited range of parameter space, which implies that it is not always clear whether the instability mechanism of the whole parameter range belongs to the same type. In this study, we investigate in more detail the dependence of the instability on the various flow parameters, including the friction velocity of air, surface tension of water, viscosities of air and water and wavelength of the unstable wave.

\section{MATHEMATICAL FORMULATION}

\section{The orr-sommerfeld equation}

We consider the growth (or decay) of a twodimensional, periodic perturbation within a viscous and incompressible air-water coupled, parallel flow with a mean velocity $U_{\ell}(z)$, where $z$ is in the vertical axis with the mean air-water interface located at $z=0$ and the subscript $\ell=a$ or $w$ stands for the variable of air or water, respectively. At the interface between the air and the water, the perturbed deviation from the mean interface is

$$
\eta(x, t)=\Re(\xi \exp [i(k x-\omega t)]),
$$

where $\xi$ is the complex perturbed amplitude, $k=k_{r}+i k_{i}$ is the complex wavenumber and is the complex cyclic frequency. A temporal instability means that for a specified real wavenumber $k=k_{r}$ the temporal growth rate $\mathfrak{I}(\omega)=\omega_{i}>0$. On the other hand, a disturbance grows spatially when $\mathfrak{\Im}(k)=k_{i}<0$ for a specified real cyclic frequency $\omega=\omega_{r}$.

The velocity vector of the flow is considered to consist of the primary mean velocity and a perturbed velocity as

$$
\vec{u}_{\ell}(x, z, t)=\left[U_{\ell}(z)+u_{\ell}^{\prime}(x, z, t)\right] \hat{i}+w_{\ell}^{\prime}(x, z, t) \hat{j} .
$$

For incompressible flow the perturbed velocity can be further expressed by the complex streamfunction,

$$
\Psi_{\ell}(x, z, t)=\phi_{\ell}(z) \exp [i(k x-\omega t)]
$$

as

$$
\begin{aligned}
& u_{\ell}^{\prime}=\Re\left(\frac{\partial \Psi_{\ell}}{\partial z}\right)=\Re\left(\frac{d \phi_{\ell}}{d z} \exp [i(k x-\omega t)]\right), \\
& w_{\ell}^{\prime}=\Re\left(-\frac{\partial \Psi_{\ell}}{\partial x}\right)=\Re\left(-i k \phi_{\ell} \exp [i(k x-\omega t)]\right) .
\end{aligned}
$$

Substituting the velocity vector into the NavierStokes equations, combining the $x$ - and $y$-component equations by eliminating the pressure gradient terms, and neglecting the nonlinear perturbed terms results in the well-known Orr-Sommerfeld equations,

$$
\begin{aligned}
& i v_{\ell} \phi_{\ell}^{(i v)}+\left(U_{\ell} k-i 2 k^{2} v_{\ell}\right) \phi_{\ell}^{\prime \prime}+\left(-U_{\ell} k^{3}-U_{\ell}^{\prime \prime} k+i k^{4} v_{\ell}\right) \phi_{\ell} \\
& =\omega\left\lfloor\phi_{\ell}^{\prime \prime}-k^{2} \phi_{\ell}\right\rfloor
\end{aligned}
$$

where $v_{\ell}$ is the kinematical viscosity of air or water. The total pressure $p_{\ell}$, which is needed in deriving the interfacial normal-stress condition, can be obtained readily from the $x$ - component linearized Navier-Stokes equation as

$$
\begin{aligned}
p_{\ell} & =\rho_{\ell}\left[\left(\frac{\omega}{k}-U_{\ell}\right) \frac{d \phi_{\ell}}{d z}+\left(\frac{d U_{\ell}}{d z}\right) \phi_{\ell}\right. \\
& \left.-i \frac{v_{\ell}}{k}\left(\frac{d^{3} \phi_{\ell}}{d z^{3}}-k^{2} \frac{d \phi_{\ell}}{d z}\right)-\xi g\right] \exp [i(k x-\omega t)],
\end{aligned}
$$

where $\rho_{\ell}$ is the density of air or water and $g$ is the gravitational acceleration.

\section{Boundary conditions}

For the fourth-order differential equation (5) in both the air and the water region, four boundary conditions are required at the interface. The physical conditions that both the vertical and horizontal velocities are continuous across the interface and the balance of the normal and tangential stresses at the interface give rise to two kinematic and two dynamic conditions. For the primary mean flows, these conditions imply that

$$
U_{a}(0)=U_{w}(0) \equiv U_{0} \text { and } v_{a} U_{a}^{\prime}(0)=v_{w} U_{a}^{\prime}(0) \equiv \tau_{0},
$$

where $U_{0}$ is the interfacial mean velocity. For the 
perturbed flows, continuity of the velocities across the interface is:

$$
u_{a}(x, \eta, t)=u_{w}(x, \eta, t) \equiv u_{0}
$$

and

$$
w_{a}(x, \eta, t)=w_{a}(x, \eta, t) \equiv w_{0} .
$$

Expressing the velocities in (9) and (10) by Taylor series about $z=0$ and neglecting the nonlinear terms, leads to the linearized kinematic conditions at $z=0$ as

$$
U_{0} \phi_{w}^{\prime}-U_{w}^{\prime} \phi_{w}-U_{0} \phi_{a}^{\prime}+U_{a}^{\prime} \phi_{a}=\omega\left[\frac{1}{k} \phi_{w}^{\prime}-\frac{1}{k} \phi_{a}^{\prime}\right],
$$

and

$$
\phi_{w}-\phi_{a}=0
$$

In reaching (11), the vertical velocity at the interface $w_{a}=w_{w}$ is related to the perturbed displacement $\xi$ through

$$
w_{0}(x, \eta, t)=\frac{D \eta}{D t}=\frac{\partial \eta}{\partial t}+u_{0}(x, \eta, t) \frac{\partial \eta}{\partial x} .
$$

Linearization of (13) results in the relationship between the perturbed amplitudes of velocity and the surface displacement at $z=0$ as

$$
\xi=\frac{k \phi_{a}(0)}{\omega-k U_{0}}=\frac{k \phi_{w}(0)}{\omega-k U_{0}} .
$$

As for the dynamic interfacial conditions, the balance of stresses and surface tension requires that,

$$
\left(\mathbf{T}_{w}-\mathbf{T}_{a}\right) \cdot \hat{\boldsymbol{n}}=-\sigma \nabla \cdot \hat{\boldsymbol{n}}
$$

where $\mathbf{T}_{a}$ and $\mathbf{T}_{w}$ are the stress tensors of air and water at the interface, $\hat{\boldsymbol{n}}$ is the normal vector of the interface and $\sigma$ is the surface tension. Decomposing the stress balance equation (15) into the components along the normal and tangential direction of the interface gives rise to the normal and tangential dynamic conditions at the interface $z=\eta$ as

$$
\begin{aligned}
& -p_{w}+\frac{2 \mu_{w}}{\left(1+\eta_{x}^{2}\right)}\left[\eta_{x}^{2} \frac{\partial u_{w}}{\partial x}-\eta_{x}\left(\frac{\partial u_{w}}{\partial z}+\frac{\partial w_{w}}{\partial x}\right)+\frac{\partial w_{w}}{\partial z}\right] \\
& -p_{a}+\frac{2 \mu_{a}}{\left(1+\eta_{x}^{2}\right)}\left[\eta_{x}^{2} \frac{\partial u_{a}}{\partial x}-\eta_{x}\left(\frac{\partial u_{a}}{\partial z}+\frac{\partial w_{a}}{\partial x}\right)+\frac{\partial w_{a}}{\partial z}\right] \\
& =\frac{\eta_{x x}}{\left(1+\eta_{x}^{2}\right)^{3 / 2}} \sigma
\end{aligned}
$$

and

$$
\begin{aligned}
& \frac{\mu_{w}}{\left(1+\eta_{x}^{2}\right)}\left[-2 \eta_{x}\left(\frac{\partial u_{w}}{\partial x}-\frac{\partial w_{w}}{\partial z}\right)+\left(1-\eta_{x}^{2}\right)\left(\frac{\partial u_{w}}{\partial z}-\frac{\partial w_{w}}{\partial z}\right)\right] \\
& -\frac{\mu_{a}}{\left(1+\eta_{x}^{2}\right)}\left[-2 \eta_{x}\left(\frac{\partial u_{a}}{\partial x}-\frac{\partial w_{a}}{\partial z}\right)+\left(1-\eta_{x}^{2}\right)\left(\frac{\partial u_{a}}{\partial z}-\frac{\partial w_{a}}{\partial z}\right)\right] \\
& =0
\end{aligned}
$$

where the dynamic viscosity $\mu_{\ell}=\rho_{\ell} v_{\ell}$. Representing the velocities in terms of the streamfunction and substituting the expression of pressure (6), the linearized normal- and tangential-stress conditions at the mean interface $z=0$ are

$$
\begin{aligned}
& U_{w}^{\prime} \phi_{w}-\left(g+\frac{\sigma}{\rho_{w}} k^{2}\right) \xi-\left(U_{0}-i 3 v_{w} k\right) \phi_{w}^{\prime}-i \frac{v_{w}}{k} \phi_{w}^{\prime \prime \prime} \\
& -s U_{a}^{\prime} \phi_{a}+s g \xi+s\left(U_{0}-i 3 v_{a} k\right) \phi_{a}^{\prime}+i s \frac{v_{a}}{k} \phi_{a}^{\prime \prime \prime} \\
& =\omega\left[-\frac{1}{k} \phi_{w}^{\prime}+\frac{s}{k} \phi_{a}^{\prime}\right],
\end{aligned}
$$

and

$$
\begin{aligned}
& U_{0} \phi_{w}^{\prime \prime}-\left(U_{w}^{\prime \prime}-k^{2} U_{0}\right) \phi_{w}-r U_{0} \phi_{a}^{\prime \prime}+r\left(U_{a}^{\prime \prime}-k^{2} U_{0}\right) \phi_{a} \\
& =\omega\left[\frac{1}{k} \phi_{w}^{\prime \prime}+k \phi_{w}-\frac{r}{k} \phi_{a}^{\prime \prime}-r k \phi_{a}\right]
\end{aligned}
$$

where the density ratio $r=\mu_{a} / \mu_{w}$ and the dynamic viscosity ratio $s=\rho_{a} / \rho_{w}$.

\section{Mean velocity profiles}

The mean velocity profiles in the air and water, $U_{\ell}(z)$, are considered to be time-independent in all of the linear stability published so far. For the basic flow in the air, the linear-logarithmic profile originally proposed by Miles (1957b) has become more or less standard [(e.g., Valenzuela (1976), Kawai (1979), and van Gastel et al. (1985)]. The profile, which characterizes a turbulent boundary layer, is given by matching the linear distribution:

$$
U_{a}(z)=U_{0}+\frac{u_{* a}^{2} z}{v_{a}}
$$

in the laminar sublayer $\left(0 \leq z \leq z_{1}\right)$ with the logarithmic distribution of well-mixed turbulence region:

$$
U_{a}(z)=U_{0}+m u_{* a}+\frac{u_{* a}}{\kappa}\left(\alpha-\tan \frac{1}{2} \alpha\right),
$$

outside the sublayer $\left(0 \leq z \leq z_{1}\right)$, where the variable $\alpha$ is 
given by

$$
\alpha=\ln \left[\beta+\left(\beta^{2}+1\right)^{2}\right], \quad \beta=\frac{2 \kappa u_{*} a}{v_{a}}\left(z-z_{1}\right),
$$

where $u_{*_{a}}$ is the friction velocity of air, $\kappa \approx 0.4$ is von Karman constant, $z_{1}=m v_{a} / u_{*}$ is a measure of the thickness of the viscous sublayer with the parameter ranging from 5 to 8 . Note that both the first and second derivatives of the linear-logarithmic profile $(20,21)$ are discontinuous at the matching height $z=z_{1}$, as shown in Fig. 1 for the example of $u_{*}=15 \mathrm{~cm} \mathrm{~s}^{-1}$ and $m=5$. Wheless and Csanady (1993) claimed that such discontinuities would cause troubles in their numerical solution of the eigensystem using the compound matrix method. However, as will be reported later, we have not encountered any difficulties in our computations using the linear-logarithmic profile.

On the water side, the flow is driven by the surface stress attributed to the air flow. In the early onset of wind, the water flow still remains to be laminar and parallel. After van Gastel et al. (1985), the velocity profile of water can be approximated by an exponential distribution:

$$
U_{w}(z)=U_{0} \exp \left(\frac{\rho_{a}}{\mu_{w}} \frac{u_{* a}^{2}}{U_{0}} z\right),
$$

where $L_{S} \equiv \mu_{w} U_{0} /\left(\rho_{a} u_{* a}^{2}\right)$ is the characteristic thickness of the shear velocity profile given the air friction velocity $u_{* a}$ and the drift velocity $U_{0}$. The profile described by (22) closely resembles that observed in Kawai7 at the initial appearance of wind wavelets.

\section{NUMERICAL METHOD}

The Orr-Sommerfeld equation (5) subject to the interfacial boundary conditions (11), (12), (18), and (19) forms an eigensystem for the complex eigenvalue $\omega$ (temporal instability problem) or $k$ (spatial instability
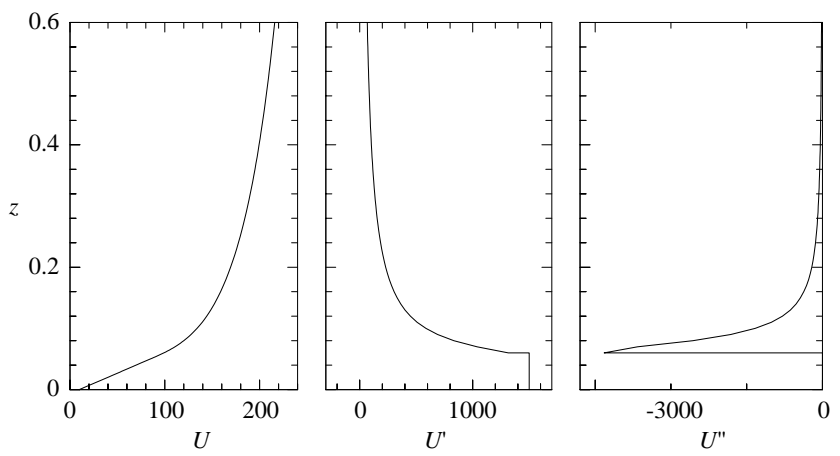

Fig. 1. Velocity profiles $U_{a}(z)$ of air and its first and second derivatives for $u_{* a}=15 \mathrm{~cm} \mathrm{~s}^{-1}$ and $\mathrm{m}=5$. problem) and the complex eigenfunctions $\phi_{a}(z)$, $\phi_{w}(z)$ and $\xi$. For a temporal instability problem, the eigensystem is linear for the complex frequency $\omega$. Whilst in a spatial instability problem, the eigenvalue system becomes nonlinear for the complex wavenumber $k$. We consider temporal growth of the wind waves in this study, and solve for the complex frequency $\omega$ for the real wavenumber $k=k_{r}$. Solution of the generalized eigenvalue problem provides the dispersion relation,

$$
\omega=\omega\left(k, r, s, \operatorname{Re}_{w}, F r, W e\right)
$$

and the corresponding eigenfunctions which determine the velocities and pressure disturbances of the instability waves. The nondimensional parameters in (23) are the Reynolds number of water flow $R e_{w}=U_{0} L_{S} / v_{\mathrm{w}}$, the Froude number $F r=U_{0}^{2} / g L_{S}$ and the Weber number $W e=U_{0}^{2} L_{S}\left(\rho_{w}-\rho_{a}\right) / \sigma$.

It is difficult to solve the eigensystem analytically, and we generally resort to numerical solution1. To integrate numerically the differential equations (5), (11), (12), (18), and (19), the complex streamfunction amplitudes $\phi_{a}(z)$ and $\phi_{w}(z)$ are discretized on the discrete grids $z=z_{k}$, and the differential operators with respect to $z$ are approximated using the second-order difference scheme.

The vertical domains of the air and water flows extend to $z= \pm \infty$. In order to make the system finite, the vertical domains in both air and water are truncated at sufficient large distances away from the interface. At the truncated boundaries $z=H_{a}$ and $-H_{w}$, the streamfunctions $\phi_{a}$ and $\phi_{w}$ are assumed to decay exponentially according to

$$
\lim _{z \rightarrow H_{a}} \phi_{a} e^{k z}=c_{a} \text { and } \lim _{z \rightarrow H_{w}} \phi_{w} e^{-k z}=c_{w},
$$

where $c_{a}$ and $c_{w}$ are constants. Accordingly, the differential terms in the discretized Orr-Sommerfeld equations at the truncated boundaries can be simplified in terms of the unknown streamfunctions within the computational domain. Different values of $H_{a}$ and $H_{w}$ have been tested in the numerical computations, and their effects on the solutions were found to be minimal.

To evaluate the second and fourth derivatives in the Orr-Sommerfeld equation at the interface, two artificial grids are extended below/above the interface for the air/water flow streamfunction. This increases the number of unknowns by four. For a grid system with $M_{a}$ and $N_{w}$ grids in the air and water regions, respectively, this leads to $M_{a}+N_{w}+5$ unknowns including the amplitude of surface deviation $\xi$ and the discrete streamfunctions $\phi_{a}\left(z_{k}\right) \equiv \phi_{k}^{a}$ and 
Table 1. Convergence property of the computed eigenvalues using different grid resolutions for the perturbed wave with wavenumber $k=1 \mathrm{~cm}^{-1}, 5 \mathrm{~cm}^{-1}$ and $9 \mathrm{~cm}^{-1}$, in a mean flow of $u_{*_{a}}=24.8 \mathrm{~cm} \mathrm{~s}^{-1}$ and $U_{0}=10.2 \mathrm{~cm} \mathrm{~s}^{-1}$

\begin{tabular}{cccc}
\hline $\begin{array}{c}\text { Grid number } \\
M_{a}=N_{w}\end{array}$ & $\begin{array}{c}k=1 \mathrm{~cm}^{-1} \\
\omega=\omega_{r}+\omega_{i} i\end{array}$ & $\begin{array}{c}k=5 \mathrm{~cm}^{-1} \\
\omega=\omega_{r}+\omega_{i} i\end{array}$ & $\begin{array}{c}k=9 \mathrm{~cm}^{-1} \\
\omega=\omega_{r}+\omega_{i} i\end{array}$ \\
\hline 100 & $32.402+1.344 i$ & $146.518+1.504 i$ & $315.527+0.552 i$ \\
300 & $33.200+0.679 i$ & $146.524+1.444 i$ & $315.559+0.528 i$ \\
500 & $33.383+0.626 i$ & $146.515+1.449 i$ & $315.566+0.524 i$ \\
700 & $33.413+0.617 i$ & $146.519+1.445 i$ & $315.569+0.524 i$ \\
900 & $33.416+0.615 i$ & $146.519+1.445 i$ & $315.570+0.524 i$ \\
\hline
\end{tabular}

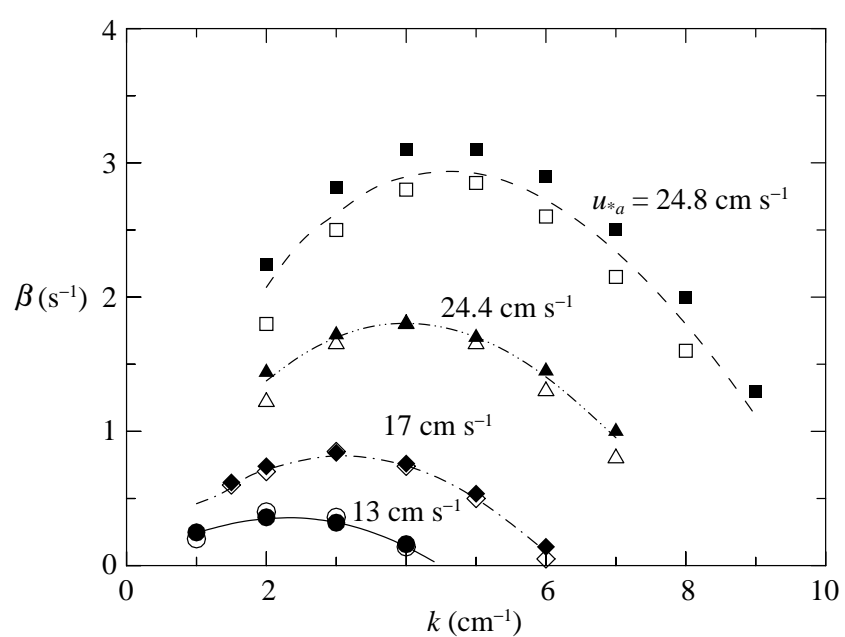

Fig. 2. Variations of the energy growth rate $\beta=2 \omega_{i}$ against the wavenumber $k$ for four values of air friction velocity $u_{*_{a}}=13$, $17,21.4$, and $24.8 \mathrm{~cm} \mathrm{~s}^{-1}$. The curves are the computed results in the present study. The solid symbols are the numerical results of Kawai (1979), ${ }^{7}$ and the open symbols are the theoretical values of van Gastel et al. $(\mathbf{1 9 8 5}){ }^{10}$

$\phi_{w}\left(z_{k}\right) \equiv \phi_{k}^{w}$. The discretized equations, including $M_{a}+$ $N_{w}+1$ discretized Orr-Sommerfeld equations and four interface boundary conditions, result in a discrete eigensystem,

$$
[\boldsymbol{A}]\{\varphi\}=\omega[\boldsymbol{B}]\{\varphi\},
$$

where $[\boldsymbol{A}]$ and $[\boldsymbol{B}]$ are the complex coefficient matrices, and the vector of eigenfunction

$$
\begin{array}{r}
\{\varphi\}=\left\{\phi_{-M_{a}}^{w}, \phi_{-M_{a}+1}^{w}, \cdots, \phi_{-1}^{w}, \phi_{0}, \phi_{2}^{w}, \xi\right. \\
\\
\left.\phi_{-2}^{a}, \phi_{-1}^{a}, \phi_{1}^{a}, \phi_{2}^{a}, \cdots, \phi_{N_{w}-1}^{a}, \phi_{N_{w}}^{a}\right\},
\end{array}
$$

with $\phi_{0}=\phi_{0}^{w}=\phi_{0}^{a}$. The equations of the complex eigensystem (25) are then solved numerically using QZ algorithm of LAPACK (1999) [1] for the complex eigenvalue $\omega$ and the vector of eigenfunction $\{\varphi\}$.

\section{RESULTS}

\section{Validation of the numerical method}

In order to validate the numerical procedures and also to determine the resolution of discretization for the calculations, we perform convergence tests by increasing the number of discrete grids. The tests are carried out for the perturbed flows with wavenumbers $k=1 \mathrm{~cm}^{-1}, 5 \mathrm{~cm}^{-1}$, and $9 \mathrm{~cm}^{-1}$ in a mean coupled flow with air friction velocity $u_{*}=24 \mathrm{~cm}$ $\mathrm{s}^{-1}$ and interfacial mean drift velocity $U_{0}=10.2 \mathrm{~cm} \mathrm{~s}^{-1}$. The case of $k=1 \mathrm{~cm}^{-1}$ is the lowest wavenumber (the longest wavelength) considered in our computations, which also requires the finest grid resolution. Whilst $k$ $=5 \mathrm{~cm}^{-1}$ corresponds to the wavenumber of the most unstable wave with the highest growth rate $\omega_{i}$. The truncated boundaries on both air and water sides locate half of the wavelength away from the interface.

The computed complex eigenvalues $\omega=\omega_{r}+i \omega_{i}$ for the test cases using different grid resolutions are shown in Table 1. For the calculations of the short $(k=$ $\left.9 \mathrm{~cm}^{-1}\right)$ and the most unstable $\left(k=5 \mathrm{~cm}^{-1}\right)$ perturbed waves, both real and imaginary parts of the computed eigenvalues converge on the third decimal place when the grid number $M_{a}=N_{w} \geq 500$. With the same grid resolution, the long wave $\left(k=1 \mathrm{~cm}^{-1}\right)$ solution can only achieve second decimal place convergence. This, however, is sufficient for the present study, and we decide to use $M_{a}=N_{w}=500$ in the following calculations.

We further validate the present numerical procedures by comparing the computed eigenvalues with the asymptotic solutions of van Gastel et al. (1985) and the numerical results obtained by Kawai (1979). Variations of the energy growth rate, $\beta \equiv 2 \omega_{i}$, with the wavenumber $k$ are showed in Fig. 2 from the present calculations as well as those by van Gastel et al. (1985) and Kawai (1979). Four air friction velocities $u_{*_{a}}=13,17,21.4$ and $24.8 \mathrm{~cm} \mathrm{~s}^{-1}$, are considered with the corresponding interfacial drift velocities of water $U_{0}=7.5,9.6,9.8$, and $10.2 \mathrm{~cm} \mathrm{~s}^{-1}$. Other parameters used are: $v_{a}=0.15 \mathrm{~cm}^{2} \mathrm{~s}^{-1}, v_{w}=0.01 \mathrm{~cm}^{2} \mathrm{~s}^{-1}, \rho_{a}=$ 
$0.0012 \mathrm{~g} \mathrm{~cm}^{-3}, \rho_{w}=1 \mathrm{~g} \mathrm{~cm}^{-3}$ and $\sigma=75 \mathrm{dyn} \mathrm{cm}^{-1}$. For low wind speeds $\left(u_{*}=13\right.$ and $\left.17 \mathrm{~cm} \mathrm{~s}^{-1}\right)$, the comparisons show good agreement between the present results and those of van Gastel et al. (1985) and Kawai (1979). Differences in the three results increase for the cases of high wind speeds $\left(u_{*}=21.4\right.$ and $\left.24.8 \mathrm{~cm} \mathrm{~s}^{-1}\right)$. The present computed growth rates are slightly lower than the numerical values of Kawai7 but higher than theoretical predictions of van Gastel et al. (1985).

Wheless and Csanady (1993) solved the same eigensystem as in the present study but used the compound matrix method as suggested by $\mathrm{Ng}$ and Reid (1979). They claimed that their computational method could not cope with the discontinuity in the second derivative of the logarithmic-linear profile $(20,21)$ used in the present and previous investigations. They therefore constructed a new smooth profile based on an integrated error function with a small second derivative. The calculated growth rates of Wheless and Csanady (1993) are almost a factor of four larger than the results of Kawai (1979). [Note that the results of Kawai (1979), which are energy growth rates, $\beta=2 k c_{i}$, were mistakenly compared with the amplitude growth rates, $k c_{i}$, by Wheless and Csanady (1993)]. Wheless and Csanady (1993) accordingly suspected that the large difference was attributed to the sensitivity of the calculations to the mean flow profiles. The quantitatively good agreement among the present results and those of Kawai (1979) and van Gastel et al. (1985), as shown in Fig. 1, however, implies that the calculations of Wheless and Csanady (1993) could be erroneous.

With the confidence in our numerical method we then focus on studying the effects of external flow variables on the instability of the perturbed wave. Specifically, the parameters we explore include the friction velocity of air $u_{*}$, the surface tension of water $\sigma$, the viscosities of air $v_{a}$ and water $v_{w}$, and the surface drift velocity $U_{0}$.

\section{Effect of the friction velocity of air}

Variations of the energy growth rate $\beta=2 \omega_{i}$ against the wavenumber $k$ for four values of air friction velocity $u_{*_{a}}=13,17,21.4$ and $24.8 \mathrm{~cm} \mathrm{~s}^{-1}$ are shown in Fig. 2. The corresponding variations in phase velocity $c=\omega_{r} /$ $k$ are plotted in Fig. 3. For any air friction velocity, there exists a wavenumber with a maximum growth rate and also a wavenumber with a minimum phase velocity. Contrary to the conclusion of Kawai (1979) and also of Wheless and Csanady (1993), the two wavenumbers do not coincide. The maximum growth rate shifts to higher wavenumber with increasing $u_{*} a$. The wavenumber at which the minimum phase velocity occurs, however, seems not to correlate with the change of $u_{*}$.
For an unstable perturbed wave with a particular wavelength $2 \pi / k$, the growth rate $\beta$ increases monotonically with the air friction velocity. This, nevertheless, is not the case for the phase velocity $c$. As shown in Fig. 3, the phase velocity increases with decreasing air friction velocity for the three values $u_{*}=17,21.4$ and $24.8 \mathrm{~cm} \mathrm{~s}^{-1}$. The phase speeds of the unstable wave of $u_{*}=13 \mathrm{~cm} \mathrm{~s}^{-1}$, however, decrease to be less than that $u_{*}$ $=17 \mathrm{~cm} \mathrm{~s}^{-1}$ of for wavenumbers below approximately 4 $\mathrm{cm}^{-1}$. This implies that there exists a critical value of $u_{*}$, below which the phase speed of an unstable wave would decrease with $u_{*}$. Such a finding has been overlooked by Wheless and Csanady (1993) as the range of $u_{* a}$ they have considered did not include the critical air friction velocity.

The unstable mode with the maximum growth rate $\beta_{\max }$ is usually the first visible wavelet under a constant wind condition as has been observed in the experiments of Kawai (1979). Kawai (1979) further showed numerically that the maximum growth rate $\beta_{\max }$ is linearly proportional to $u_{* a}^{3.5}$. The analyses of van Gastel et al. (1985) however reveal that $\beta_{\max } \propto u_{*}^{3}{ }_{a}$ for the range of friction velocities $5 \mathrm{~cm} \mathrm{~s}^{-1} \leq u_{*} \leq 40 \mathrm{~cm} \mathrm{~s}^{-1}$. The variation of the present numerical $\beta_{\max }$ with $u_{*}$ is shown in Fig. 4 in logarithmic-scale coordinate. Power fitting of the data is also plotted. The result indicates that there indeed exists a simple functional relation between $\beta_{\max }$ and $u_{*}$, and the relation is the same as that obtained by Kawai (1979): $\beta_{\max } \approx u_{* a}^{3.5}$.

\section{Effect of the viscosities of air and water}

To understand the effects of fluid viscosities on the initial development of the perturbed waves, variations of the growth rate and phase speed with the

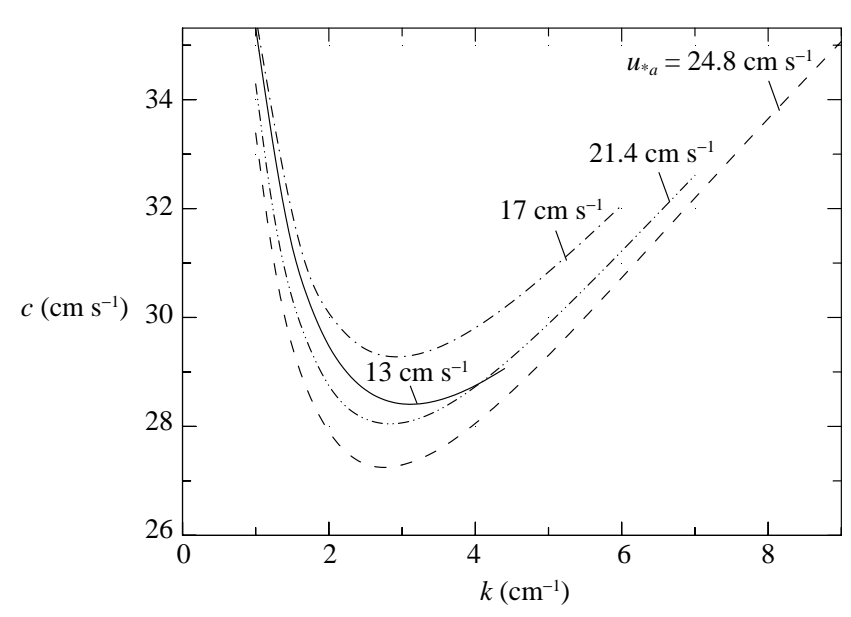

Fig. 3. Variations of the phase velocity $c=\omega_{r} / k$ against the wavenumber $k$ for four values of air friction velocity $u_{* a}=13,17,21.4$, and $24.8 \mathrm{~cm} \mathrm{~s}^{-1}$. 
wavenumber for different viscosities of air and water are shown in Fig. 5 and 6, respectively. The three kinematical viscosities, $v_{a}=0.12,0.15,0.18 \mathrm{~cm}^{2} \mathrm{~s}^{-1}$ and $v_{w}=0.017,0.01,0.005 \mathrm{~cm}^{2} \mathrm{~s}^{-1}$, correspond to the values at low, normal and high temperatures, respectively. Reducing the air viscosity would increase the growth rate, but the impact is minor (Fig. 5). The phase speed of the unstable wavelet is virtually unaffected by the kinematical viscosity of air. In contrast, the growth rate increases substantially when the viscosity of water decreases (Fig. 6). Significant shift in the wavenumber of the maximum growth rate $\beta_{\max }$ is also observed when varying the water viscosity. Accompanying the abrupt increase in the growth rate with

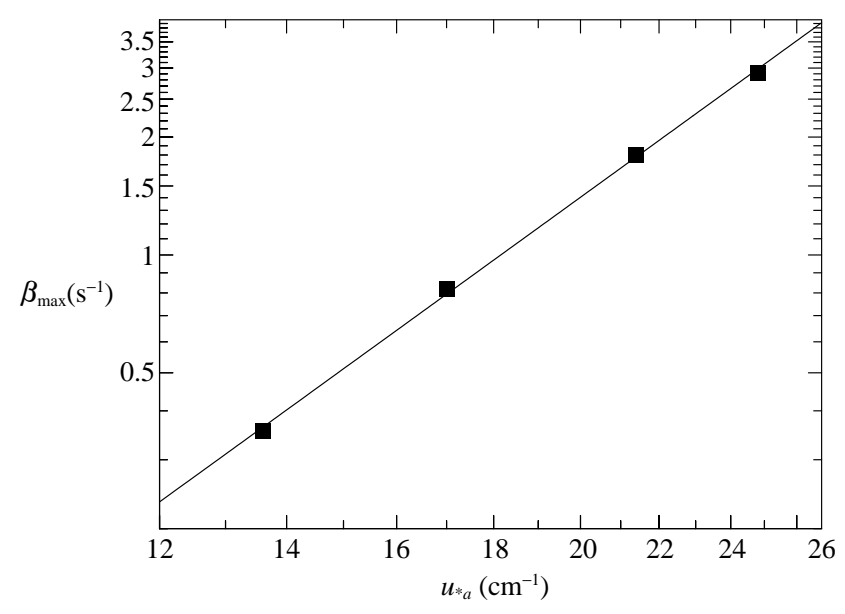

Fig. 4. Variation of the maximum growth rate $\beta_{\max }$ with the air friction velocity $u_{* a}$. The square symbols are the numerical results, and the line is the least-square fitting.

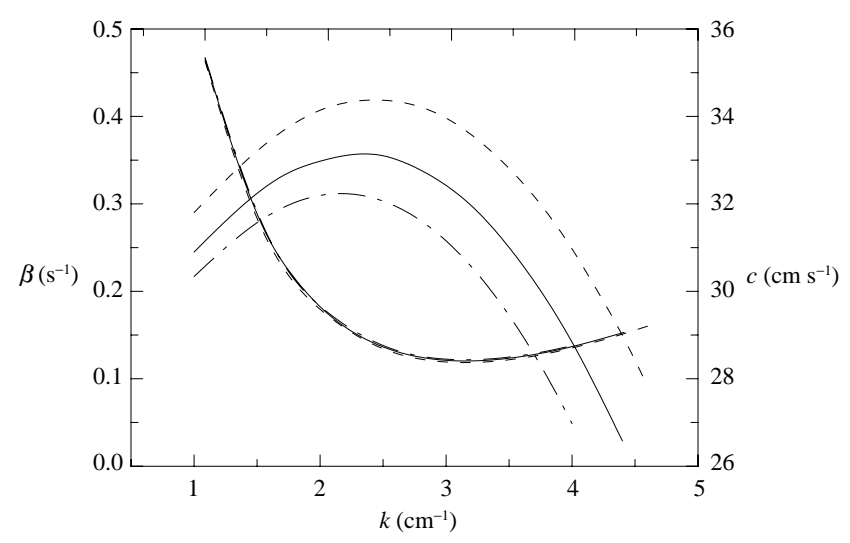

Fig. 5. Variations of the growth rate $\beta=2 \omega_{i}$ and phase speed $c=\omega_{r} / k$ with the wavenumber $k$ for different values of air viscosity: $v_{a}$ $=0.12 \mathrm{~cm}^{2} \mathrm{~s}^{-1}$ (dashed curves), $0.15 \mathrm{~cm}^{2} \mathrm{~s}^{-1}$ (solid curves), and $0.18 \mathrm{~cm}^{2} \mathrm{~s}^{-1}$ (dash-dot curves). The air friction velocity $\boldsymbol{u}_{*_{a}}=$ $13 \mathrm{~cm} \mathrm{~s}^{-1}$. reducing water viscosity, the phase speed of the unstable wave decreases drastically. Such a feature becomes more significant for waves of high wavenumbers or short wavelengths.

\section{Effect of the surface tension of water}

The surface tension at an air-water interface is affected by the temperature and salinity of water, and also by the presence of surface-active materials (surfactants). The presence of salts in water increases the intermolecular attraction and the surface tension of seawater is therefore slightly greater than that of fresh water at the same temperature. Increasing the water temperature, on the other hand, reduces the surface tension by weakening the molecular attraction. The presence of surfactant films on the water surface also decreases the surface tension significantly, and the induced "Plateau-Marangoni-Gibbs effect" consequently dampens the surface waves generation as show by Gottiferedi and Jameson (1968), Creamer and Wright (1992), and Saetra (1998). To study the impact of surface tension on the wind wave generation, three surface tensions, $\sigma=75$ and $70 \mathrm{dyn}^{-1}$, corresponding to the values at low $\left(\approx 2{ }^{\circ} \mathrm{C}\right)$, normal $\left(\approx 20^{\circ} \mathrm{C}\right)$ and high $\left(\approx 56^{\circ} \mathrm{C}\right)$ water temperatures, are used to perform the stability analyses. Fig. 7 shows the variations of the growth rate and phase speed with the wavenumber for the three values of surface tension. Reducing the surface tension increases the growth rate and decreases the phase velocity of the instability waves. However, very minor effects of the surface tension on the initial wavelets are observed for the large range of temperaturesurface tension dependence considered.

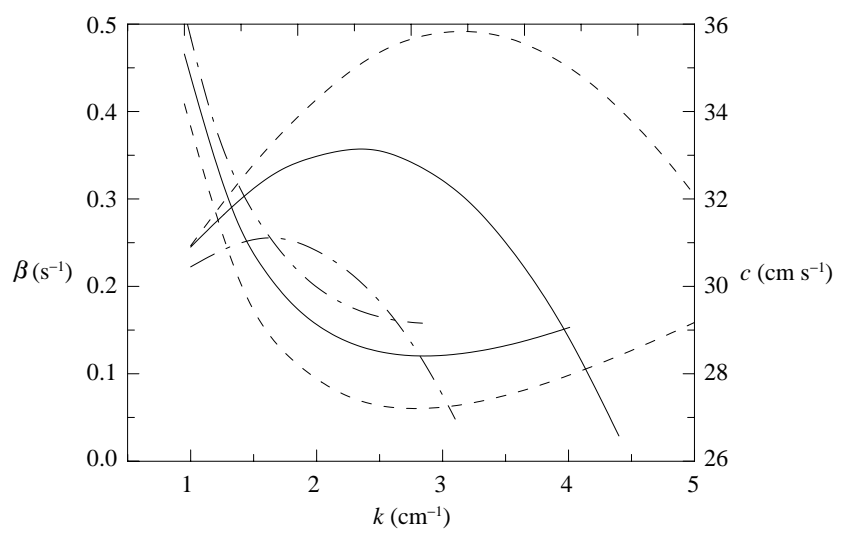

Fig. 6. Variations of the growth rate $\beta=2 \omega_{i}$ and phase speed $c=\omega_{\mathrm{r}} / k$ with the wavenumber $\boldsymbol{k}$ for different values of viscosity of water: $v_{w}=0.005 \mathrm{~cm}^{2} \mathrm{~s}^{-1}$ (dashed curves), $0.01 \mathrm{~cm}^{2} \mathrm{~s}^{-1}$ (solid curves), and $0.017 \mathrm{~cm}^{2} \mathrm{~s}^{-1}$ (dash-dot curves). The air friction velocity $u_{* a}=13 \mathrm{~cm} \mathrm{~s}^{-1}$. 


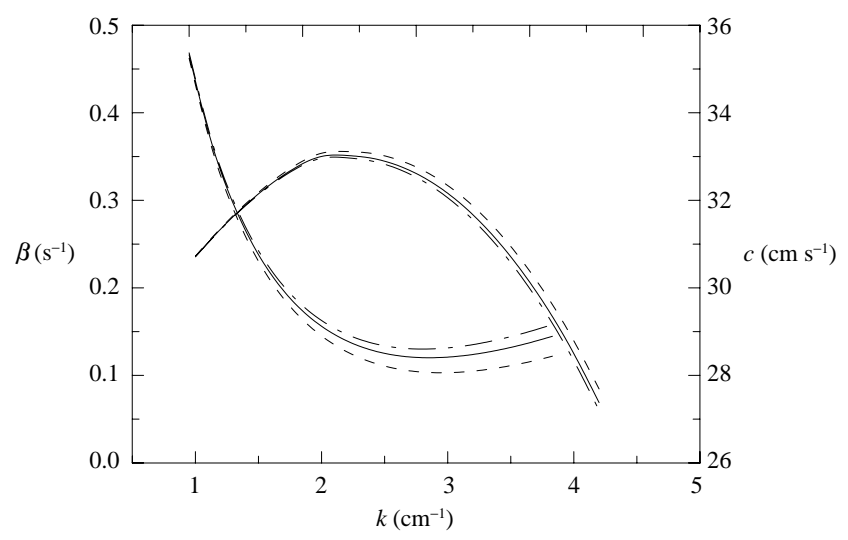

Fig. 7. Variations of the growth rate $\beta=2 \omega_{i}$ and phase speed $c=\omega_{r} / k$ with the wavenumber $k$ for different values of surface tensions: $\sigma=78 \mathrm{dyn} \mathrm{cm}^{-1}$ (dash-dot curves), $75 \mathrm{dyn}^{-1}$ (solid curves), and $70 \mathrm{dyn} \mathrm{cm}^{-1}$ (dashed curves). The air friction velocity $\boldsymbol{u}_{* a}$ $=13 \mathrm{~cm} \mathrm{~s}^{-1}$.

\section{CONCLUDING REMARKS}

The instability of a perturbed wave in an air-water coupled shear flow is analyzed with the application to the generation of initial wavelets at the air-sea interface. An efficient and robust numerical method is developed for such a purpose. Comparison of the computed growth rates of instability waves with previous numerical and asymptotic analyses validates the accuracy of the present method. The numerical procedure is then applied to explore the impact of the flow parameters on the initial development of wind-generated waves. The major conclusions drawn from this study are:

(1) There exist a maximum growth rate and a minimum phase velocity for the range of the wavenumber of instability waves. The maximum growth rate $\beta_{\max }$ of an instability wave increases with the air friction velocity $u_{* a}$, and is linearly proportional to $u_{*}^{3.5}$. There is no apparent correlation between the maximum growth rate and the minimum phase velocity wavenumber.

(2) Reducing the air viscosity only slightly raises the growth rate of an instability wave, but the phase speed is virtually unaffected. In contrast, the growth rate increases substantially when the viscosity of water decreases. Significant shift in the wavenumber of the maximum growth rate $\beta_{\max }$ is also observed when varying the water viscosity.

(3) For the surface tension varying with a wide range of temperature $\left[O\left(1^{\circ} \mathrm{C}\right)\right.$ to $\left.O\left(10^{2 \circ} \mathrm{C}\right)\right]$, the impact on the generation of instability wave is minor.

All of the theoretical models concerning the initial wind-generated waves so far are based on the analysis of the temporal growth of the instability waves. Recent experiment by Caulliez et al. (1998), however, showed that the scales of the most spatially amplified waves could differ from the scales of the most temporally amplified waves. This implies that the convective instability mechanism might also play an important role as the temporal instability. The major difficulty in analyzing the spatial instability arises from the need to solve a system of nonlinear equations for the complex eigenvalue of wavenumber. This difficulty, however, can be resolved by the searching procedure outlined in Lin and Chen (1998) and utilizing the present efficient numerical implementation for temporal stability analysis. Such a numerical procedure for spatial instability analysis of the air-water coupled flow is under current development.

\section{ACKNOWLEDGMENTS}

This work was supported by grants from the National Science Council of Taiwan (NSC 91-2611-M008-002 and 92-2611-M-008-003).

\section{REFERENCES}

1. Anderson, E., Bai, Z., Bischof, C., Blackford, S., Demmel, J., Dongarra, J., Du Croz, J., Greenbaum, A., Hammarling, S., McKenney, A., Ostrouchov, S., and Sorensen, D., LAPACK Users' Guide, 3rd Edition, SIAM, Philadelphia, PA, (1999).

2. Benjamin, T.B., "Shearing Flow Over a Wavy Boundary," J. Fluid Mech., Vol. 6, pp. 161-205 (1959).

3. Caulliez, G., Ricci, N., and Dupont, R., "The Generation of the First Visible Wind Waves," Phys. Fluids, Vol. 10, pp. 757-759 (1998).

4. Creamer, D.B. and Wright, J.A., "Surface Films and Wind Wave Growth," J. Geophys. Res., Vol. 97, pp. 5221-5229 (1992).

5. Gottiferedi, J.C. and Jameson, G.J., "The Suppression of Wind-Generated Waves by a Surface Film," J. Fluid Mech., Vol. 32, pp. 609-618 (1968).

6. Kawai, S., "Generation of Initial Wavelets by Instability of a Coupled Shear Flow and Their Evolution to Wind Waves," J. Fluid Mech., Vol. 93, pp. 661-703 (1979).

7. Lin, S.P. and Chen, J.N., "Role Played by the Interfacial Shear in the Instability Mechanism of a Viscous Liquid Jet Surrounded by a Viscous Gas in a Pipe," $J$. Fluid Mech., Vol. 378, pp. 37-51 (1998).

8. Lock, R.C., "Hydrodynamic Stability of the Flow in the Laminar Boundary Layer Between Parallel Streams," Proc. Camb. Phil. Soc., Vol. 50, pp. 105-124 (1954).

9. Miles, J. W., "On the Generation of Surface Waves by Shear Flows," J. Fluid Mech., Vol. 3, pp. 185-204 (1957a).

10. Miles, J.W., "On the Velocity Profile for Turbulent Flow 
Near a Small Wall," J. Aero. Sci., Vol. 24, p. 704 (1957b).

11. Miles, J.W., "On the Generation of Surface Waves by Shear Flows. Part 4," J. Fluid Mech., Vol. 13, pp. 433448 (1962).

12. Ng, B.S. and Reid, W.H., "An Initial Value Method for Eigenvalue Problems Using Compound Matrices," $J$. Comput. Phys., Vol. 30, pp. 125-136 (1979).

13. Phillips, O.M., "On the Generation of Waves by Turbulent Wind," J. Fluid Mech., Vol. 2, pp. 417-445 (1957).

14. Saetra, O., "Effects of Surface Film on the Linear Stability of an Air-Sea Interface," J. Fluid Mech., Vol. 357, pp.
59-81 (1998)

15. Valenzuela, G.R., "The Growth of Gravity-Capillary Waves in a Coupled Shear Flow," J. Fluid Mech., Vol. 76, pp. 229-250 (1976).

16. van Gastel, K., Janssen, P.A., and Komen, G.J., "On Phase Velocity and Growth Rate of Wind-Induced Gravity-Capillary Waves," J. Fluid Mech. Vol. 161, pp. 199-216 (1985).

17. Wheless, G.H. and Csanady, G.T., "Instability Waves on the Air-Sea Interface," J. Fluid Mech. Vol. 248, pp. 363-381 (1993). 